\title{
Cyclophilin A: a possible host modulator in Chandipura virus infection
}

\author{
Daya V. Pavitrakar ${ }^{1} \cdot$ Nitin M. Atre ${ }^{1} \cdot$ Anuradha S. Tripathy $^{1} \cdot$ Pratip Shil $^{1}$ (i) \\ Received: 6 April 2021 / Accepted: 28 July 2021 / Published online: 17 September 2021 \\ (c) The Author(s), under exclusive licence to Springer-Verlag GmbH Austria, part of Springer Nature 2021
}

\begin{abstract}
Chandipura virus (CHPV), belonging to the genus Vesiculovirus of the family Rhabdoviridae, has been identified as one of the causes of pediatric encephalitis in India. Currently, neither vaccines nor therapeutic drugs are available against this agent. Considering that the disease progresses very fast with a high mortality rate, working towards the development of potential therapeutics against it will have a public health impact. Although the use of viral inhibitors as antiviral agents is the most common way to curb virus replication, the mutation-prone nature of viruses results in the development of resistance to antiviral agents. The recent development of proteomic platforms for analysis of purified viral agents has allowed certain upregulated host proteins that are involved in the morphogenesis and replication of viruses to be identified. Thus, the alternative approach of inhibition of host proteins involved in the regulation of virus replication could be explored for their therapeutic effectiveness. In the current study, we have evaluated the effect of inhibition of cyclophilin A (CypA), an immunophilin with peptidyl-prolyl cis/trans-isomerase activity, on the replication of CHPV. Treatment with cyclosporin A, used in vitro for the inhibition of CypA, resulted in a 3-log reduction in CHPV titer and an undetectable level of CypA in comparison to an untreated control. An in silico analysis of the interaction of the CHPV nucleoprotein with the human CypA protein showed stable interaction in molecular docking and molecular dynamics simulations. Overall, the results of this study suggest a possible role of CypA in facilitating CHPV replication, thus making it one of the potential host factors to be explored in future antiviral studies.
\end{abstract}

\section{Introduction}

Chandipura virus (CHPV), a negative-sense RNA virus, belongs to the genus Vesiculovirus of the family Rhabdoviridae [1]. The virus was first isolated in the village of Chandipur in the Nagpur district of Maharashtra state, India, in 1965 [2]. Later, in the year 2003, large encephalitis outbreaks were reported in the states of Andhra Pradesh, Telangana, and Maharashtra [3]. The disease was clinically characterized by a high-grade fever of short duration, vomiting, altered sensorium, generalized convulsions, and decerebrate posture, leading to grade IV coma and death within $48 \mathrm{~h}$ of hospitalization [3]. The CHPV genome encodes five proteins, namely, nucleocapsid protein $\mathrm{N}$, phosphoprotein $\mathrm{P}$, matrix protein $\mathrm{M}$, glycoprotein $\mathrm{G}$, and large protein $\mathrm{L}$. These

Handling Editor: William G Dundon.

Pratip Shil

shil.p@gov.in; shilpratip@gmail.com

1 ICMR-National Institute of Virology, 130/1 Sus Road, Pashan, Pune 411021, India proteins interact with host cell machinery to enable the various stages of the viral life cycle, beginning with entry and uncoating of the virus, followed by transcription and replication of its genome, and finally the assembly and release of progeny virions [4].

The role of different host proteins in the virus life cycle has been studied for different viral infections. Recently, with the help of computational approaches based on structure and sequence similarity, 121 putative host interactors were identified for CHPV [5]. One of the host proteins, cyclophilin A (CypA), which has been demonstrated to be associated with the life cycle of several viruses, including VSV, the prototype virus of the genus Vesiculovirus [6]. CypA is an immunophilin with peptidyl-prolyl cis/trans isomerase (PPIase) activity that plays a key role in protein folding [7]. It is also associated with cellular functions such as cell signalling, transcriptional regulation, protein folding, and trafficking $[8,9]$ besides playing an important role as a receptor for the immunosuppressive drug cyclosporin A (CsA) [10]. Dawar et al. [11] reviewed its diverse roles in infections with viruses including hepatitis $B$ virus (HBV), vaccinia virus (VV), human immunodeficiency virus type 1 (HIV-1), 
hepatitis $\mathrm{C}$ virus (HCV), coronaviruses (CoVs), vesicular stomatitis virus (VSV), human cytomegalovirus (HCMV), flaviviruses, and enterovirus 71. An antiviral role of CypA has been demonstrated against vesicular stomatitis virus (VSV) [6], a related virus belonging to the genus Vesiculovirus of the family Rhabdoviridae. The recent observations by Zhang et al. [12] on proteomic profiling of purified rabies virus particles have also documented the association of CypA with virus particles, emphasizing its possible role in virus replication. The cyclophilins are known for their high binding affinity for CsA [10]. Thus, inhibition of CypA using known inhibitors such as CsA and its synthetic analogs (NIM811, alisporivir) [11] may pave the way for exploring the role of CypA in viral infections. The availability of cyclosporin A and its analogs for clinical use makes it easier to target this particular host protein to curtail viral infection, thus making it one of the attractive targets for antiviral therapy [13]. Owing to the diverse role of CypA in viral infections, the aim of the current study was to explore the role of CypA in CHPV infection using in silico as well in vitro approaches.

\section{Materials and methods}

\section{Study design}

Chandipura virus strain 034627 (CIN0327) was used for the study. To investigate the involvement of CypA in CHPV infection, CsA was used as an inhibitor of CypA. The cell cytotoxicity of CsA in RD cells was determined using an MTT assay. Concentrations at which $>90 \%$ cell viability was observed were selected for further analysis. To assess the effect of CypA inhibition on CHPV replication, a plaque reduction assay was carried out. The growth kinetics of CHPV in RD cells in the presence and absence of CypA inhibitor were compared. The presence of extracellular CypA during CHPV infection in the presence or absence of CsA was determined using a commercially available CypA ELISA. The experimental work was further supported by in silico studies. The 3D structure of the $\mathrm{N}$ protein of $\mathrm{CHPV}$ 034627 was predicted and validated. The validated structure was then analyzed for its interaction with human cyclophilin A by in silico docking. The stability of the docked complex was analyzed by molecular dynamics simulation.

\section{Virus and cells}

A CHPV strain isolated during the 2003 outbreak, CHPV034627 [3], maintained at the lowest passage level, was used for all in vitro experiments, including plaque reduction assays and growth kinetics assays carried out in this study. The RD cell line (a human rhabdomyosarcoma cell line) was procured from ATCC (ATCC CCL-136, USA). It was maintained in Dulbecco's modified Eagle's medium (DMEM) (Sigma, USA) supplemented with $10 \%$ fetal bovine serum (FBS, Invitrogen, USA) and $100 \mathrm{U}$ of penicillin and $100 \mu \mathrm{g}$ of streptomycin (Invitrogen, USA) per $\mathrm{ml}$ at $37{ }^{\circ} \mathrm{C}$ in a $5 \%$ $\mathrm{CO}_{2}$ incubator for propagation of the virus and growth kinetics experiments.

\section{Virus stock preparation and quantitation}

RD cells were infected with CHPV 034627 at a multiplicity of infection (moi) of 0.01 for $1 \mathrm{~h}$ at $37^{\circ} \mathrm{C}$. The unabsorbed virus was removed, and DMEM with $2 \%$ FBS was added. The culture supernatant was harvested when $80-90 \%$ of cells showed a cytopathic effect (CPE). This material was further clarified by centrifugation and stored at $-80^{\circ} \mathrm{C}$ for further use.

The quantitation of the reference virus stock in plaqueforming units was carried out in RD cells using a plaque assay as described earlier [14]. The assay was terminated at $24 \mathrm{~h}$ postinfection, and plaques were counted manually. The virus titer was expressed as plaque-forming units/ml (PFU/ $\mathrm{ml}$ ) and was used to determine the moi in further infections and experimental growth kinetics assays.

\section{Inhibition of CypA}

To determine the inhibitory concentrations of CsA, the 50\% cytotoxic concentration $\left(\mathrm{CC}_{50}\right)$ of commercially available CsA (CypA inhibitor) was first determined using an MTT assay carried out in RD cells with CsA concentrations in the range of 5-500 $\mu \mathrm{M}$ in quadruplicate as described earlier [15].

\section{Effect of inhibition of cyclophilin A (CypA) with cyclosporin A (CsA) on CHPV replication}

Based on the results of the MTT assay, non-toxic concentrations (> 90\% cell viability) of CsA were chosen for studying the effect of inhibition of CypA on CHPV replication. In a preliminary experiment to assess the effect of CypA inhibition on CHPV replication, a plaque reduction assay in RD cells was carried out using $100 \mathrm{PFU}$ of CHPV and $5 \mu \mathrm{M}$ and $10 \mu \mathrm{M}$ concentrations of CsA in $1.8 \% \mathrm{CMC}$ overlay medium after virus adsorption. Wells overlaid with CMC overlay after virus infection served as a positive control, and uninfected wells overlaid with CMC overlay served as a negative control.

The effect of CypA inhibition on CHPV replication was assessed by comparing the growth kinetics of CHPV in RD cells in the presence or absence of CsA. In these experiments, cells were infected with CHPV at an moi of 0.01 and $5 \mu \mathrm{M}$ or $10 \mu \mathrm{M}$ concentrations of CsA were used. Uninfected cells suspended in CsA-containing medium and in medium 
Fig. 1 Growth kinetics of CHPV in the presence of the cyclophilin A inhibitor cyclosporin A

\section{Effect of CypA inhibition on CHPV growth kinetics}

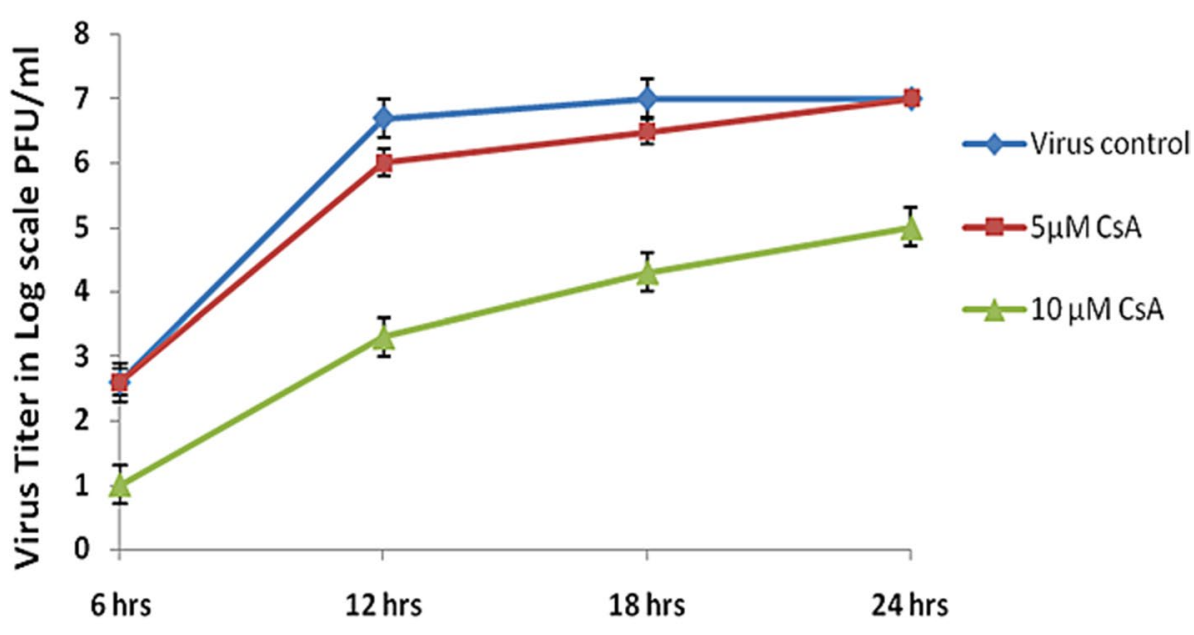

Time post infection without CsA served as a drug control and a negative control, respectively, whereas cells infected with the virus and suspended in medium without CsA served as a virus control. The culture supernatant was harvested at 6, 12, 18, and $24 \mathrm{~h}$ postinfection and stored at $-80^{\circ} \mathrm{C}$ for further virus titer determination by plaque assay.

In order to investigate the association of CypA inhibition with CHPV replication kinetics, we measured extracellular and intracellular levels of CypA in uninfected and infected cells in the presence and absence of CsA.

For extracellular detection of CypA, culture supernatant harvested at different time points was tested by ELISA. For intracellular detection of CypA, cells were collected from each well in RIPA lysis buffer (Cell Signalling Technology, USA) containing protease inhibitor cocktail. Cell lysis was carried out for $10 \mathrm{~min}$ on ice, followed by centrifugation at $14,000 \mathrm{rpm}$ for $10 \mathrm{~min}$. The clarified cell lysate was then used for measurement of CypA levels by ELISA. The level of CypA was analyzed in triplicate using a commercially available ELISA kit (Human Cyclophilin A ELISA, Sigma, USA) following the manufacturer's instructions.

\section{D structure prediction and molecular docking}

The 3D structure of the N protein of CHPV strain 034627 (GenBank Protein ID AD063665) was predicted using a Swiss-Model online workstation [16] using the vesicular stomatitis virus (VSV) $\mathrm{N}$ protein as a template (PDB ID: 2GIC) [17]. The predicted structure was analyzed for the quality of modeling using the PROCHECK tool available at http://www.ebi.ac.uk/thornton-srv/databases/pdbsum/Gener ate.html [18]. The 3D structure of the human CypA protein was retrieved from the Protein Data Bank (PDB ID: 1CWA).
Visualizations of protein structure and images were generated using Discovery Studio Visualizer.

The 3D structure of human CypA (1CWA.pdb) retrieved from the RCSB Protein Data Bank (PDB) was used for docking with the predicted structure of the $\mathrm{N}$ protein of $\mathrm{CHPV}$ using the PATCHDOCK server (Molecular Docking Algorithm Based on Shape Complementarity Principles) (https:// bioinfo3d.cs.tau.ac.il/PatchDock/https://bioinfo3d.cs.tau.ac. il/PatchDock/) [19]. The top 10 docking complexes generated by PATCHDOCK were refined using the FireDock tool (Fast Interaction Refinement in molecular DOCKing) available on the PATCHDOCK server. The refined complex with the lowest binding energy was selected for studying the stability of interaction using molecular dynamics simulation. The binding affinity of the selected complex was then calculated using PRODIGY (PROtein binDIng enerGY prediction) tool available online at https://bianca.science.uu.nl/ prodigy/ [20].

\section{Molecular dynamics simulation}

The stability of the docked complex of the CHPV N protein with the human CypA protein was evaluated by molecular dynamics simulation using the GROMACS-2020.2 molecular dynamics package [21]. The bonded and non-bonded interactions between the protein molecules were analyzed using the OPLS-AA/L all-atom force field [22]. The system was equilibrated, and charge neutralization was carried out by adding suitable ions as per established protocols. The system pressure and temperature were equilibrated at 1 bar and $300^{\circ} \mathrm{K}$ for an equilibration period of $100 \mathrm{ps}$. The MD simulations were set for $10 \mathrm{~ns}$, and the RMSD was determined to obtain a stable protein-protein complex [23]. 
Fig. 2 (a) Extracellular levels of cyclophilin A during CHPV replication. (b) Intracellular levels of cyclophilin A during CHPV replication

\section{a}

Study of presence of extracellular Cyclophilin A in CHPV infected cells with and without Cyclosporin treatment

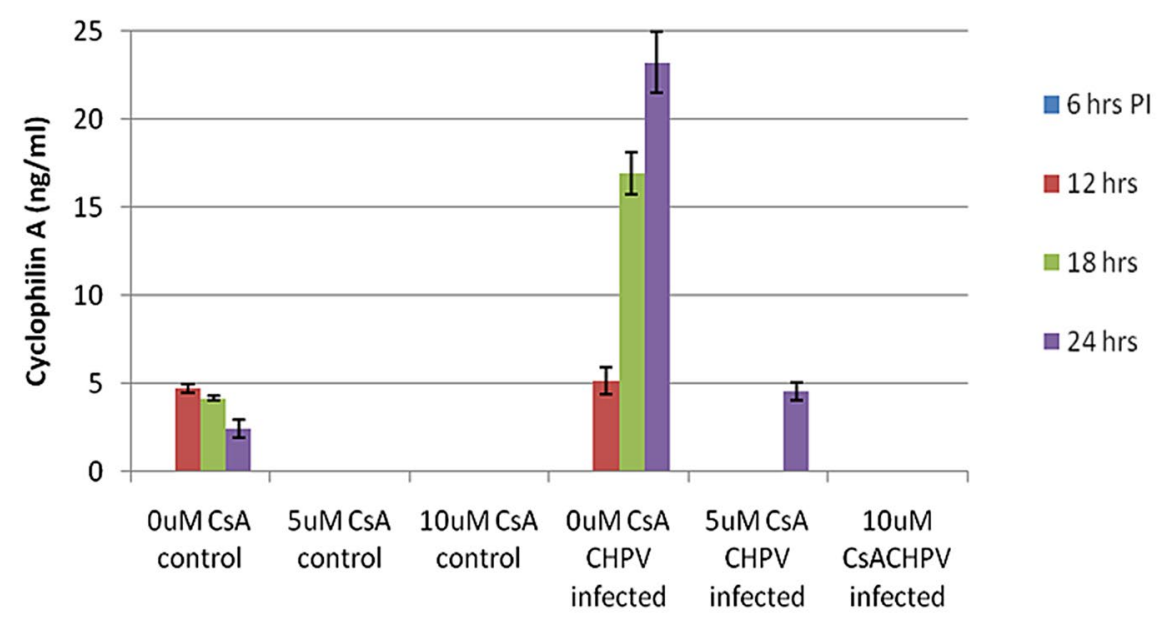

Treatment groups

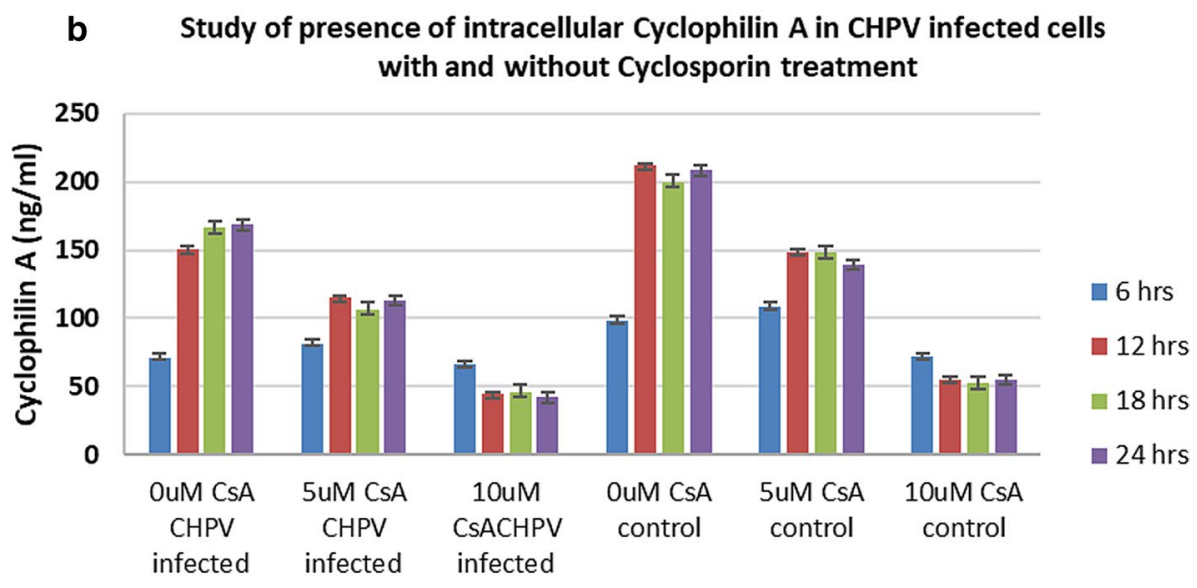

\section{Results}

\section{Virus titration}

Using a plaque assay, the titer of the virus was found to be $1 \times 10^{7} \mathrm{PFU} / \mathrm{ml}$. This reference stock was used to calculate the moi for the growth kinetics assay (Supplementary Fig. S1).

\section{Inhibition of CypA}

Using an MTT assay to analyze the toxic effects of the CypA inhibitor CsA on RD cells, the $\mathrm{CC}_{50}$ was found to be $40 \mu \mathrm{M}$. The non-toxic concentrations of $\operatorname{CsA}(5 \mu \mathrm{M}$ and $10 \mu \mathrm{M})$ with $>90 \%$ cell viability were used in the inhibition study. A plaque reduction assay showed a fourfold reduction in the number of plaques (mean plaque number \pm SD: $24 \pm 5$ ) in cells treated with $10 \mu \mathrm{M}$ CsA (SD: $100 \pm 8$ ) compared to those treated with $5 \mu \mathrm{M}$ CsA $(100 \pm 7)$ or untreated (Supplementary Fig. S2).

The growth kinetics of CHPV in the presence of CsA showed a dose-dependent effect on virus replication (Fig. 1). The infection of RD cells reached a titer of $1 \times 10^{7} \mathrm{PFU} / \mathrm{ml}$ (mean $\pm 1 \mathrm{SE}, \log _{10} \mathrm{PFU} / \mathrm{ml}$ ) in the untreated virus control cells and cells treated with $5 \mu \mathrm{M}$ CsA, but it reached only $1 \times 10^{5} \mathrm{PFU} / \mathrm{ml}\left(\right.$ mean $\left.\pm 1 \mathrm{SE}, \log _{10} \mathrm{PFU} / \mathrm{ml}\right)$ in the presence of $10 \mu \mathrm{M}$ CsA. Figure 1 shows the effect of inhibition of CypA on CHPV replication. A reduction in virus titer by $>\log 3\left(\right.$ mean $\pm 1 \mathrm{SE}, \log _{10} \mathrm{PFU} / \mathrm{ml} ; 2 \times 10^{4} \mathrm{PFU} / \mathrm{ml} \mathrm{vs}$. $1 \times 10^{7} \mathrm{PFU} / \mathrm{ml}$ in the virus control and $3 \times 10^{6} \mathrm{PFU} / \mathrm{ml}$ in $5-\mu \mathrm{M}$-CsA-treated cells) was observed at $18 \mathrm{~h}$ postinfection in cells treated with $10 \mu \mathrm{M} \mathrm{CsA}$. The impairment of replication of CHPV observed after inhibition of CypA indicated a possible role of this protein in virus replication. 
Fig. 3 The docked complex of the CHPV N protein with the human cyclophilin A molecule

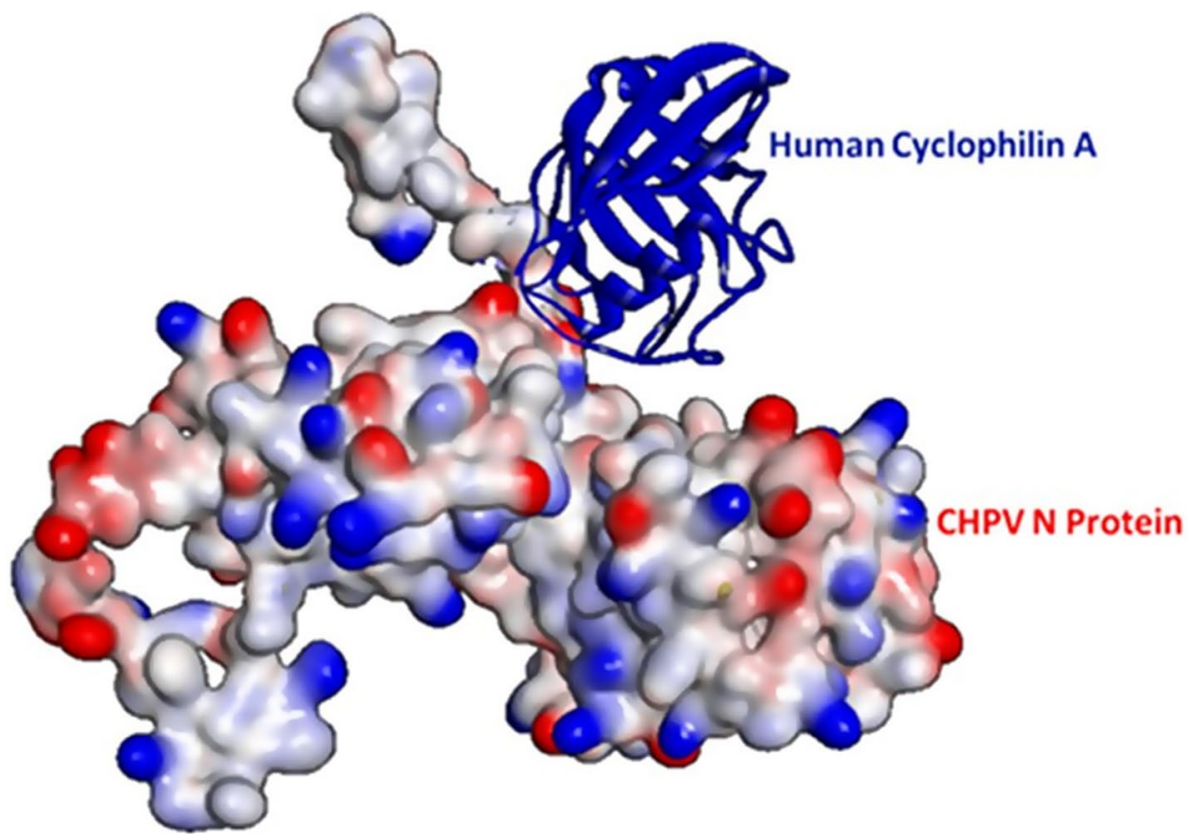

Furthermore, measurement of CypA levels in the culture supernatants harvested for the viral growth kinetics experiment revealed the association of the extracellular level of CypA with that of viral egress. The cells treated with CypA inhibitor after virus infection showed lower levels (decrease of $3 \operatorname{logs}$ ) of both the virus and CypA in the culture supernatant at different time points, whereas the untreated virusinfected cells showed a substantial increase in CypA concentration at 18 and $24 \mathrm{~h}$ postinfection. In the case of the uninfected drug control cells treated with $5 \mu \mathrm{M}$ and $10 \mu \mathrm{M}$ CsA, the CypA was not detectable, whereas a slight increase in CypA was detected in control cells (Fig. 2a).

The assays for intracellular detection of CypA revealed an approximately fourfold reduction in the CypA level in the presence of $10 \mu \mathrm{M}$ CsA in both the CHPV-infected and uninfected cells (Fig. 2b).

Analysis of the virus multiplication rate as a function of time using the Mann-Whitney test showed a significant difference in growth kinetics of CHPV in CsA-treated $(10 \mu \mathrm{M})$ vs. untreated RD cells $(p<0.01)$.

\section{D structure prediction and molecular docking}

The 3D structure of the N protein of CHPV-034627 was predicted using the VSV N protein as a template (PDB ID: 2GIC). Assessment of the Ramachandran plot revealed that the overall percentage of residues in favored and allowed regions was $99.5 \%$, indicating the reliability of the modeled structure. The in silico molecular docking of the predicted $\mathrm{N}$ protein structure with Human CypA (PDB ID: 1CWA) was retrieved from the RCSB Protein Data Bank (PDB). The top-ten-ranking complexes generated by PatchDock were subjected to refinement using the FireDock tool. The docked complex refined by FireDock with a binding energy in solution of $-3.19 \mathrm{kcal} / \mathrm{mol}$ formed one hydrogen bond between Ala20 of the N protein and Glu120 of cyclophilin A. The van der Waals energy component was the principal stabilizing energy for their interactions, as calculated by FireDock. The best solution is shown in Figure 3. As determined using the online PRODIGY tool, the binding energy of the selected complex was $\Delta \mathrm{G}=-8.5 \mathrm{kcal} /$ mol. After FireDock refinement, the selected docked complex with the lowest binding energy was used to analyze the stability of the interaction in a molecular dynamics simulation.

\section{Molecular dynamics}

To evaluate the stability of the CHPV N protein complexed with human CypA, a molecular dynamics simulation was performed using GROMACS-2020.2. The temperature and pressure were equilibrated by applying the NVT and NPT ensemble, respectively, with a period of 100 ps (Fig. 4a and $b$ ). The minimized potential energy was estimated to be $-3.717 \times 10^{6} \mathrm{~kJ} / \mathrm{mol}$ for the docked protein complex. The RMSD plot of the complex is shown in Figure 4c. The RMSD gradually increased from $0.2 \mathrm{~nm}$ to $0.6 \mathrm{~nm}$ after $6 \mathrm{~ns}$ and then stabilized further to $\sim 0.7 \mathrm{~nm}$ after $7 \mathrm{~ns}$. The RMSF plot shown in Figure 4d indicated fluctuations in the CHPV N protein, covering the amino acid stretch from residues 9 to 26 . Fluctuation in the human CypA protein was observed in the amino acid stretch from residues 100 to 125 . It should be noted that these regions of maximum fluctuation form the binding interface in the complex with H-bonds formed between Ala 20 of the N protein and Glu 120 of CypA. These findings 

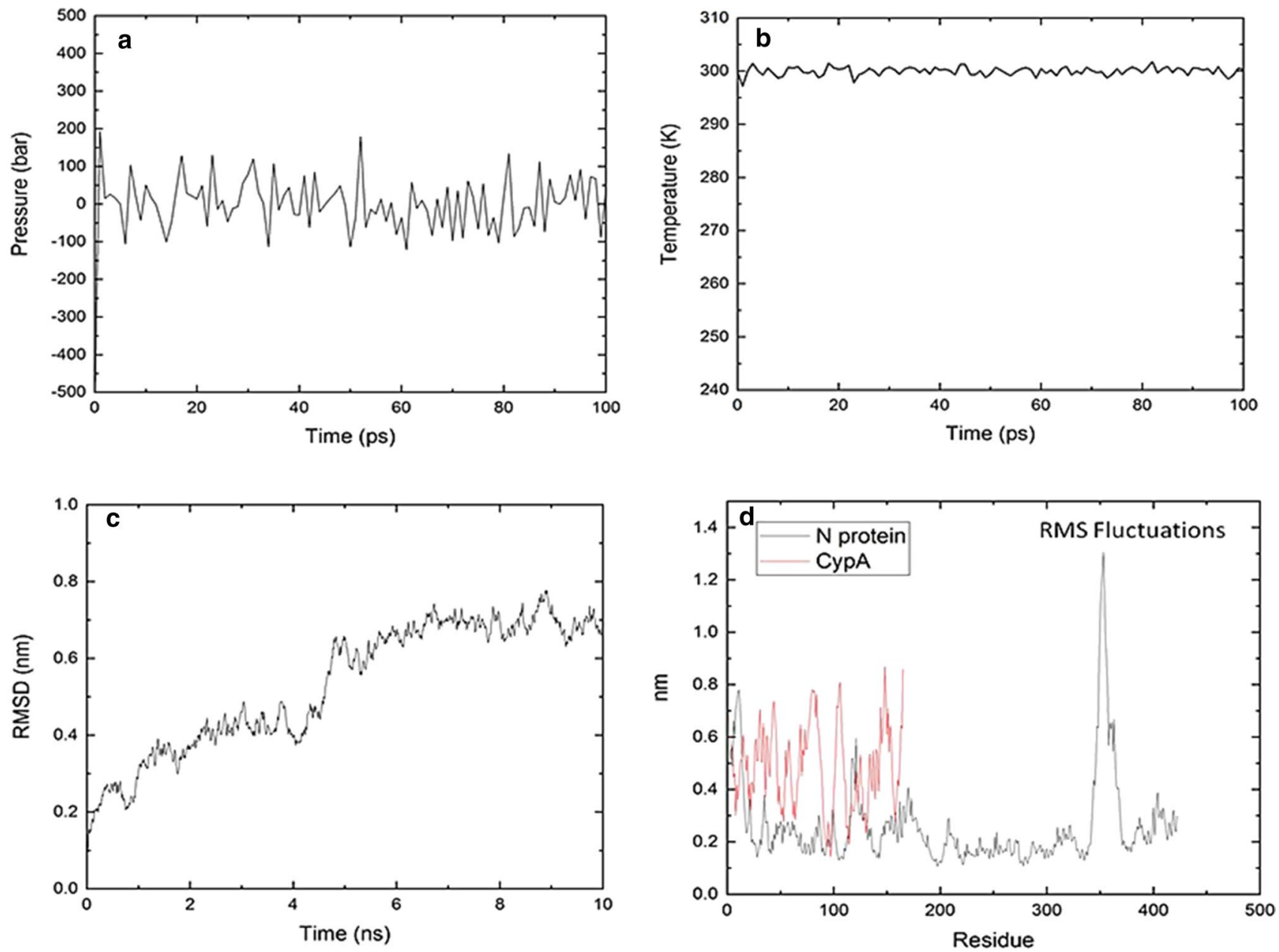

Fig. 4 (a-d) Molecular dynamics simulation results for the CHPV N protein complexed with human CypA. (a) Plot of pressure vs. time. (b) Plot of temperature vs. time. (c) Root mean square deviation

(RMSD) plot of the CHPV N protein complexed with human CypA. (d) Root mean square fluctuation (RMSF) plot representing the fluctuations in the side-chain residues for both the proteins

demonstrate the flexibility and stability of the docked protein complex.

\section{Discussion}

Like many other viruses, CHPV encodes few proteins of its own. Consequently, concerted interactions between host and viral proteins are essential for efficient virus replication [24]. Earlier studies carried out on vesicular stomatitis virus demonstrated the role of CypA as a chaperone protein and one of the cellular factors required for VSV replication. Bose et al. concluded that the interaction of CypA with the VSV $\mathrm{N}$ protein enables the folding of nucleocapsids into a transcriptionally competent conformation required for optimal transcription efficiency of the VSV-NJ genome [6].

In the present study, we evaluated the role of CypA in CHPV replication. We first analyzed the in silico interaction

of the CHPV N protein with the human CypA protein. In the absence of a known structure of the CHPV N protein, the 3D structure was predicted using the VSV N protein as a template. The predicted structure was judged to be stable and reliable by PROCHECK analysis (Ramachandran plot). Molecular docking of the predicted structure of the CHPV $\mathrm{N}$ protein with human CypA showed stable binding. MD simulations indicate that the complex is highly stable.

In vitro experiments indicated elevated extracellular levels of CypA in CHPV-infected cells at 18-24 h postinfection (Fig. 2a), and the virus titer was $1 \times 10^{7} \mathrm{PFU} / \mathrm{ml}$ at $18 \mathrm{~h}$ postinfection (Fig. 1). These findings indicate that an increased level of extracellular CypA may be associated with cytopathic effects. The effect of CsA-induced inhibition of CypA was observed in viral growth kinetics experiments. A greater than three-log reduction in virus titer was observed at $18 \mathrm{~h}$ postinfection when infected cells were treated with $10 \mu \mathrm{M}$ CsA. 
We have also conducted laboratory experiments for the determination of intracellular levels of CypA. It was observed that treatment of both uninfected cells and cells infected with CHPV with $10 \mu \mathrm{M}$ CsA (a CypA inhibitor) resulted in a fourfold reduction in CypA levels.

Drawing an analogy from reports on the $\mathrm{N}$ protein of VSV, a virus related to CHPV, it could be concluded that the host CypA may play a similar role in the replication of CHPV. In parallel, in silico studies indicated stable binding of the CHPV N protein with the host CypA, thus supporting our in vitro observations. However, experimental work to study the interaction of CypA with the CHPV N protein needs to be performed to test the accuracy of the in silico predictions.

For the first time, we have obtained evidence for a possible role of the host CypA protein in the inhibition of CHPV replication. The information obtained in the present study can be further evaluated using different nonimmunosuppressive analogs of CsA with conserved CypA inhibition potential.

Supplementary information The online version contains supplementary material available at https://doi.org/10.1007/s00705-021-05237-1.

Acknowledgements The authors would like to thank Dr. Priya Abraham, Director ICMR-National Institute of Virology, for her encouragement and meaningful input.

Funding The present study was supported by institutional funding of the National Institute of Virology, Pune.

\section{Declarations}

Conflict of interest The authors declare that they have no conflict of interest.

\section{References}

1. Fenner F (1976) Classification and nomenclature of viruses. Second report of the International Committee on Taxonomy of Viruses. Intervirology 7(1-2):1-115. https://doi.org/10.1159/ 000149938

2. Bhatt PN, Rodrigues FM (1967) Chandipura: a new Arbovirus isolated in India from patients with febrile illness. Indian J Med Res 55:1295-1305

3. Rao BL, Basu A, Wairagkar NS, Gore MM, Arankalle VA, Thakare JP, Jadi RS, Rao KA, Mishra AC (2004) A large outbreak of acute encephalitis with high fatality rate in children in Andhra Pradesh, India, in 2003, associated with Chandipura virus. Lancet 364(9437):869-874. https://doi.org/10.1016/ S0140-6736(04)16982-1

4. Banerjee AK (1987) Transcription and replication of rhabdoviruses. Microbiol Rev 51:66-87

5. Rajasekharan S, Rana J, Gulati S, Sharma SK, Gupta V, Gupta $S$ (2013) Predicting the host protein interactors of Chandipura virus using a structural similarity-based approach. Pathog Dis 69:29-35. https://doi.org/10.1111/2049-632X.12064
6. Bose S, Mathur M, Bates P, Joshi N, Banerjee AK (2003) Requirement for cyclophilin A for the replication of vesicular stomatitis virus New Jersey serotype. J Gen Virol 84:16871699. https://doi.org/10.1099/vir.0.19074-0

7. Wang P, Heitman J (2005) The cyclophilins. Genome Biol 6:226. https://doi.org/10.1186/gb-2005-6-7-226

8. Liao CH, Kuang YQ, Liu HL, Zheng YT, Su B (2007) A novel fusion gene, TRIM5-Cyclophilin A in the pig-tailed macaque determines its susceptibility to HIV-1 infection. AIDS 21(Suppl 8):S19-S26. https://doi.org/10.1097/01.aids.0000304692. 09143.1b

9. Nigro P, Pompilio G, Capogrossi MC (2013) Cyclophilin A: a key player for human disease. Cell Death Dis 4:e888. https:// doi.org/10.1038/cddis.2013.410

10. Göthel SF, Marahiel MA (1999) Peptidyl-prolyl cis-trans isomerases, a superfamily of ubiquitous folding catalyst. Cell Mol Life Sci 55:423-436. https://doi.org/10.1007/s000180050 299

11. Dawar FU, Tu J, Khattak MN, Mei J, Lin L (2017) Cyclophilin A: a key factor in virus replication and potential target for antiviral therapy. Curr Issues Mol Biol 21:1-20. https://doi.org/10. 21775/cimb.021.001

12. Zhang Y, Wang Y, Feng Y, Tu Z, Lou Z, Tu C (2020) Proteomic profiling of purified rabies virus particles. Virol Sin 35:143155. https://doi.org/10.1007/s12250-019-00157-6

13. Fu J, Tjandra M, Becker C, Bednarczyk D, Capparelli M, Elling R, Hanna I, Fujimoto R, Furegati M, Karur S, Kasprzyk T, Knapp M, Leung K, Li X, Lu P, Mergo W, Miault C, Ng S, Parker D et al (2014) Potent nonimmunosuppressive cyclophilin inhibitors with improved pharmaceutical properties and decreased transporter inhibition. J Med Chem 57:8503-8516. https://doi.org/10.1021/jm500862r

14. Pavitrakar DV, Ayachit VM, Mundhra S, Bondre VP (2015) Development and characterization of reverse genetics system for the Indian West Nile virus lineage 1 strain 68856. J Virol Methods 226:31-39. https://doi.org/10.1016/j.jviromet.2015.09. 008

15. Kumar P, Nagarajan A, Uchil PD (2018) Analysis of cell viability by the MTT assay. Cold Spring Harb Protoc. https://doi.org/10. 1101/pdb.prot095505

16. Waterhouse A, Bertoni M, Bienert S, Studer G, Tauriello G, Gumienny R, Heer FT, de Beer T, Rempfer C, Bordoli L, Lepore R, Schwede T (2018) SWISS-MODEL: homology modelling of protein structures and complexes. Nucleic Acids Res 46:W296W303. https://doi.org/10.1093/nar/gky427

17. Green TJ, Zhang X, Wertz GW, Luo M (2006) Structure of the vesicular stomatitis virus nucleoprotein-RNA complex. Science 313:357-360. https://doi.org/10.1126/science.1126953

18. Laskowski RA, Hutchinson EG, Michie AD, Wallace AC, Jones ML, Thornton JM (1997) PDBsum: a Web-based database of summaries and analyses of all PDB structures. Trends Biochem Sci 22:488-490. https://doi.org/10.1016/s0968-0004(97)01140-7

19. Schneidman-Duhovny D, Inbar Y, Nussinov R, Wolfson HJ (2005) PatchDock and SymmDock: servers for rigid and symmetric docking. Nucleic Acids Res 33:W363-W367. https://doi.org/10.1093/ nar/gki481

20. Xue LC, Rodrigues JP, Kastritis PL, Bonvin AM, Vangone A (2016) PRODIGY: a web server for predicting the binding affinity of protein-protein complexes. Bioinformatics 32:3676-3678. https://doi.org/10.1093/bioinformatics/btw514

21. Abraham MJ, Murtola T, Schulz R, Páll S, Smith JC, Hess B, Lindahl E (2015) GROMACS: high performance molecular simulations through multi-level parallelism from laptops to supercomputers. SoftwareX 1-2:19-25. https://doi.org/10.1016/j.softx. 2015.06.001 
22. Kaminski GA, Friesner RA, Tirado-Rives J, Jorgensen WL (2001) Evaluation and reparametrization of the OPLS-AA force field for proteins via comparison with accurate quantum chemical calculations on peptides. J Phys Chem B 2:6474-6487. https://doi.org/ 10.1021/jp003919d

23. Shukla R, Shukla H, Kalita P, Sonkar A, Pandey T, Singh DB, Kumar A, Tripathi T (2018) Identification of potential inhibitors of Fasciola gigantica thioredoxin1: computational screening, molecular dynamics simulation, and binding free energy studies. J Biomol Struct Dyn 36:2147-2162. https://doi.org/10.1080/ 07391102.2017.1344141
24. Guleria A, Kiranmayi M, Sreejith R, Kumar K, Sharma SK, Gupta S (2011) Reviewing host proteins of Rhabdoviridae: possible leads for lesser studied viruses. J Biosci 36:929-937. https://doi. org/10.1007/s12038-011-9164-4

Publisher's Note Springer Nature remains neutral with regard to jurisdictional claims in published maps and institutional affiliations. 